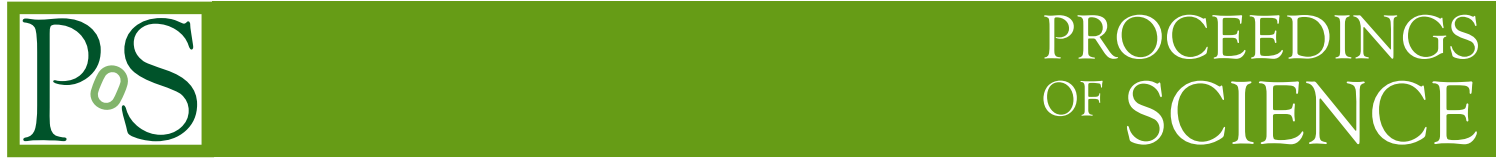

\title{
Commissioning and Performance of the CMS Silicon Pixel Tracker Detector
}

\author{
Gavril Giurgiu*i \\ Johns Hopkins University \\ E-mail: ggiurgiuefnal.gov
}

The innermost tracking system of the CMS experiment is the pixel detector. The pixel detector consists of 3 barrel layers and two endcap disks at each barrel end. There are a total of 66 million pixel channels. The size of each pixel is $100 \times 150 \mu \mathrm{m}^{2}$. The pixel detector is designed to exploit the charge sharing among adjacent pixels to gain hit resolution down to $12 \mu \mathrm{m}$. Detector calibration results as well as results from cosmic ray studies with the CMS pixel detector are presented.

RD09 - 9th International Conference on Large Scale Applications and Radiation Hardness of Semiconductor Detectors

30 September - 2 October 2009

Florence, Italy

${ }^{*}$ Speaker.

† On behalf of the CMS collaboration 


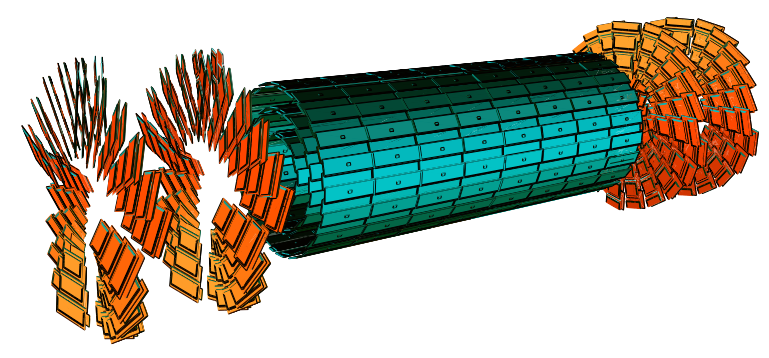

Figure 1: The CMS pixel detector. The three barrel layers are shown in blue and the two forward disks at each barrel end are shown in red.

\section{Introduction}

The Compact Muon Solenoid (CMS) experiment [1] at the Large Hadron Collider [2] has a tracking system which is entirely silicon based and is composed of two main sub-detectors. The innermost tracking detector is segmented in two dimensions. The smaller sensitive unit is called a pixel. The pixel detector is surrounded by the silicon strip detector which is segmented in only one dimension.

The silicon pixel detector [1] is crucial for the reconstruction of charged particle trajectories. Accurate determination of the trajectory parameters is important for finding tracks with large impact parameter and for reconstructing decay vertices which are displaced with respect to the primary interaction point. For example, the impact parameter resolution as function of the track pseudo-rapidity, for $10 \mathrm{GeV} / \mathrm{c}^{2}$ muons is between 20 and 30 microns and it reaches about $10 \mathrm{mi}$ crons for $100 \mathrm{GeV} / \mathrm{c}^{2}$ muons [3]. Both displaced tracks and displaced vertices are characteristic of the decay of long lived $B$ hadrons. Identification of $B$ hadron decays, known as $B$ tagging is important in many measurements involving bottom and top quarks as well as in searches for the Higgs boson or searches for physics beyond the standard model (SM).

The pixel detector consists of three barrel layers and two disks at each barrel end as seen in figure 1. The three barrel layers have radii of 4.4, 7.3 and $10.2 \mathrm{~cm}$, respectively. Each layer is composed of rectangular detector units called modules. Eight modules along the global $\mathrm{Z}$ direction form a ladder. A full size detector unit contains 66,560 pixel channels which are read out by sixteen read out chips (ROC) [4]. There are 52 x $80=4160$ pixels per ROC. A half size detector unit contains 33,280 pixels read out by eight ROCs. There are a total of 48 million pixel channels in the pixel barrel detector. Pixels are connected to the ROCs using the bump bonding technique. The forward disks are located at $z= \pm 34.5 \mathrm{~cm}$ and $z= \pm 46.5 \mathrm{~cm}$ from the interaction region. The forward disks extend radially from 6 to $15 \mathrm{~cm}$. Each disk is divided in half-disks. There are 12 blades in a half-disk. The blades are tilted by 20 degrees in a turbine-like geometry to enhance charge sharing between pixels due to the induced Lorentz drift and to larger average incident angle of tracks with respect to the normal incidence. There are 4230 ROCs corresponding to about 18 million pixel channels in the pixel forward detector.

Charged particles passing through the silicon sensors produce electron-hole pairs by ionizing 

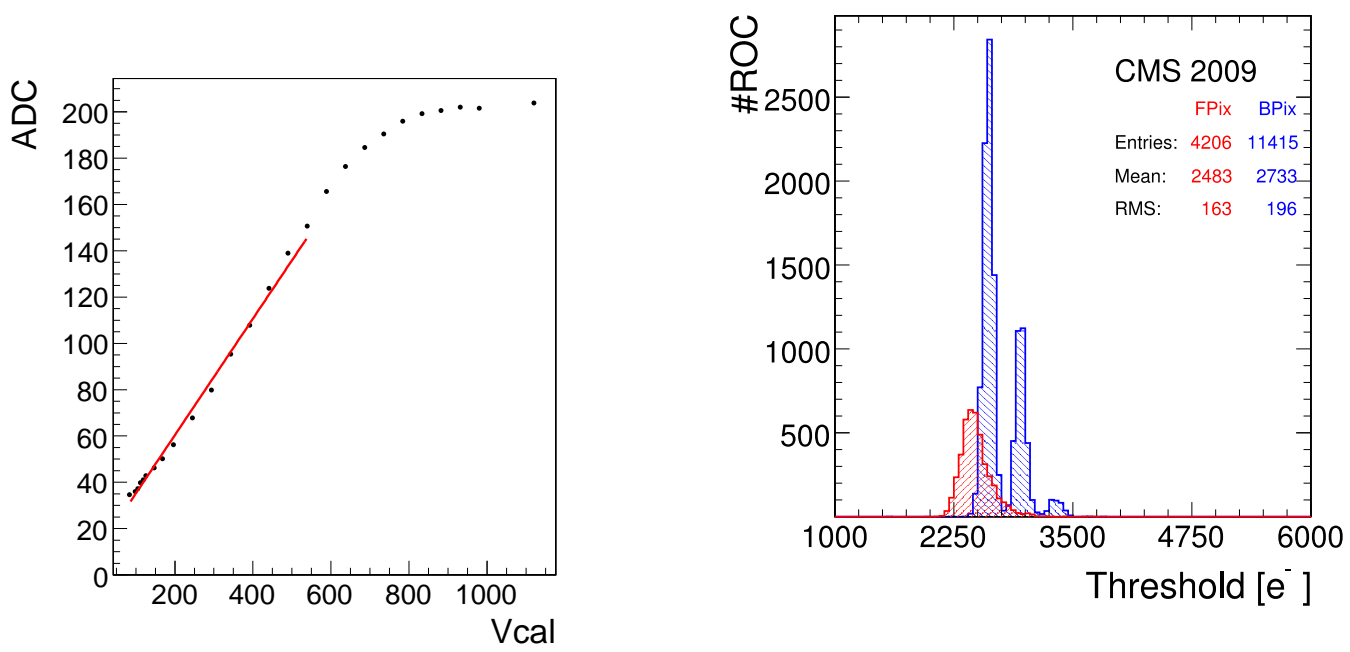

Figure 2: Left: The black points show the dependence of the pixel detector response in ADC counts as function of the injected charge in ADC counts. A saturation plateau is reached at a level of 45,000 electrons which is twice the most probable MIP charge deposition. The solid red line shows a linear approximation fit. Right: Distributions of the pixel thresholds for barrel detector (blue) and for the forward detector (red).

silicon atoms. A bias voltage is applied on the silicon sensors and the freed electrons are collected at the pixel electrodes. The pixel sensor is approximately $280 \mu \mathrm{m}$ thick. Each pixel has dimensions of $100 \mu \mathrm{m}$ in the $r \phi$ direction and $150 \mu \mathrm{m}$ in the $z$ direction. The pixel size is driven by the readout circuit area required for each pixel. The collected ionization charge is, in general, shared among a few pixels. Charge sharing allows position interpolation and leads to improved position resolution. In the barrel detector the charge sharing in the $r \phi$ direction is enhanced by the Lorentz drift of the ionization charge in the 3.8 Tesla magnetic field of the CMS magnetic solenoid.

In order to improve spatial resolution, the pixel detector is placed very close to the interaction region. Being close to the colliding beams, the pixel detector will be subject to heavy irradiation. The three barrel layers will be exposed to particle fluences of $4 \times 10^{14} \mathrm{n}_{e q} / \mathrm{cm}^{2} /$ year, 1.2 x $10^{14} \mathrm{n}_{e q} / \mathrm{cm}^{2} /$ year and $0.6 \times 10^{14} \mathrm{n}_{e q} / \mathrm{cm}^{2} /$ year, respectively. Irradiation exposure will also be different along the $z$ direction. Regions closer to the interaction region will be exposed to more radiation than regions farther away. All components of the pixel detector are specified to stay operational up to a particle fluence of at least $6 \times 10^{14} \mathrm{n}_{e q} / \mathrm{cm}^{2}$. As the pixel detector suffers radiation damage and the ionization charge is partly trapped in the sensor defects, charge collection become non-uniform. Charge that has to travel longer distances to reach the pixel electrodes will be collected less efficiently due to trapping effects. Increased bias voltage will reduce the charge loss due to trapping. The pixel hit reconstruction software is designed to account for irradiation effects and correct for biases induced by charge collection inefficiencies. The reconstruction software relies on frequent calibrations like the measurement of the Lorentz drift or the charge collection inefficiency.

\section{Detector Calibration}

Various detector calibrations are needed to extract the track hit position starting from the 

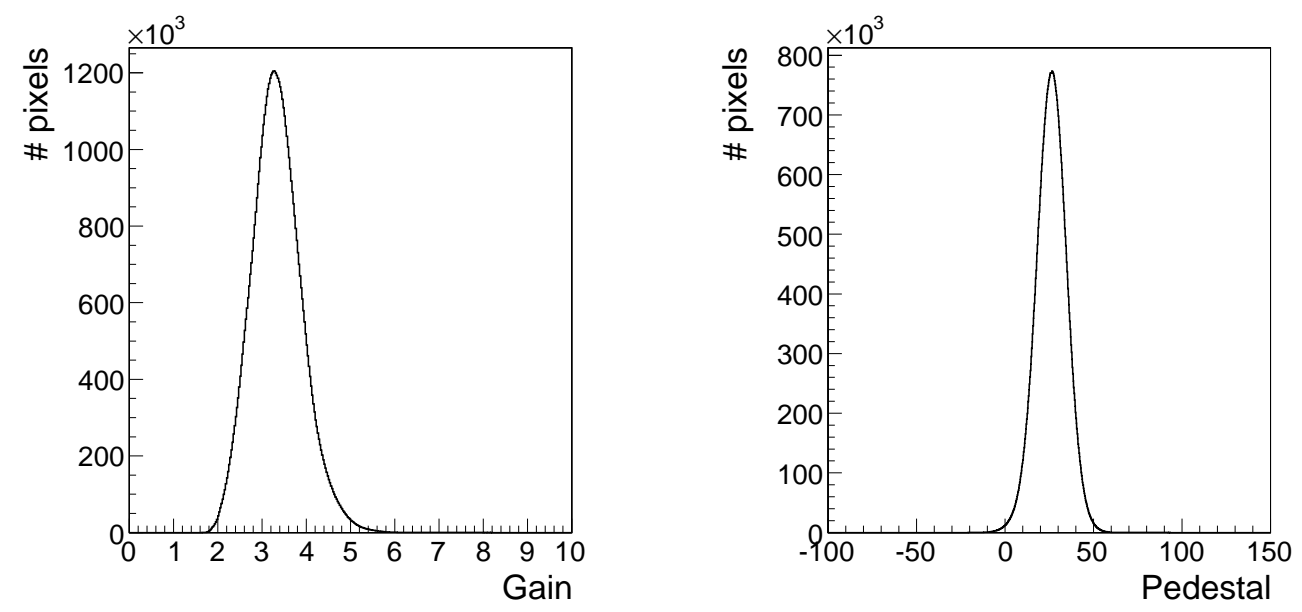

Figure 3: Distributions of gains and pedestals of all pixels in barrel and forward detectors combined.

charge collected by each pixel. We describe here the calibration needed to transform the charge from ADC counts to electrons and the measurement of the pixel readout thresholds.

\subsection{Charge Calibration}

The charge collected by each pixel is digitized and recorded in ADC counts. To transform the charge from ADC counts to electrons, the following calibration procedure is used. A known amount of charge is injected in each pixel. The injected charge is varied and the pixel response in ADC counts is recorded. The amount of charge injected in each pixel is controlled by an 8 bit DAC (VCAL) setting at ROC level. The covered charge range is between $\approx 10^{2}$ and $\approx 10^{5}$ electrons. The calibration of the internally injected charge was performed using $\mathrm{x}$-ray sources of known energies. The relation between charge in electrons and charge in VCAL units was found $\mathrm{Q}$ (electrons) $=65.5 \times \mathrm{Q}(\mathrm{VCAL}$ units $)$ - 414. The pixel-to-pixel variation on the slope parameter has an RMS of $\approx 9$ while the offset parameter has an RMS of $\approx 570$ electrons. These variations induce a $15 \%$ systematic uncertainty on the charge injection scale, which in turn leads to a degradation of the pixel hit resolution by up to $20 \%$ in certain regions of the track angular acceptance. It is planned to calibrate the charge detector response with collision tracks to improve the hit resolution.

The dependence of the recorded charge in ADC counts as function of VCAL units is shown in figure 2. Since the detector response is approximately linear up to 45,000 electrons the ADC vs $\mathrm{VCAl}$ dependence is fit with a linear function. The inverse of the slope is called gain and the offset parameter is called pedestal. The mean and RMS of the gain and pedestal distributions for the barrel and forward detectors are shown in figure 3 and summarized in Table 1. Finally, the charge in electrons as function of the charge in $\mathrm{ADC}$ counts is given by:

$\mathrm{Q}$ (electrons) $=65.5 \times[\mathrm{Q}(\mathrm{ADC}$ counts $)-$ pedestal $] /$ gain -414 electrons. Dedicated charge calibration runs determine the gain and pedestal for each pixel.

\subsection{Readout Pixel Thresholds}

The charge recorded by each pixel is read out only if the charge is above the pixel threshold. Zero suppression is required to reduced the event data size. The thresholds are adjustable at pixel 


\begin{tabular}{c|c|c} 
& barrel & forward \\
\hline gain mean & 3.5 & 2.9 \\
gain RMS & 0.5 & 0.5 \\
\hline pedestal mean & 25.5 & 28.0 \\
pedestal RMS & 8.1 & 9.5 \\
\hline threshold mean & 2733 & 2483 \\
threshold RMS & 196 & 163
\end{tabular}

Table 1: Mean and RMS of gain, pedestal and threshold distributions for the barrel and forward detectors.

level. They are measured using "S-curve" scans of the efficiency versus injected charge. The injected charge is controlled by the same VCAL parameter used for charge injection in the gain calibration runs. The pixel efficiency is defined as the fraction of times the injected charge is read out. The dependence of the pixel efficiency as function of injected charge in VCAL units is fit with a Gauss error function. The parameters of the error function measure the pixel threshold and the pixel noise. The threshold distributions for the barrel and for the forward detectors is shown in figure 2 .

Thresholds are minimized for each ROC using a highly iterative procedure. In barrel about $33 \%$ of ROCs are set at higher thresholds with respect to the rest of the ROCs. The higher threshold ROCs correspond to the second and third peaks of the barrel threshold distribution. Work will be done in the near future to lower all thresholds in the barrel at the $\approx 2,500$ electron level. A summary of the mean and RMS spread of the pixel thresholds is given in Table 1. The intrinsic front-end ROC noise is also determined from the "S-curve" fit and found to be of the order of 100-200 electrons. The rest of the readout chain contributes with an additional noise of about 300 electrons. These noise levels are very small compared to the pixel thresholds of $\approx 2,500$ electrons and with the mean charge of $\approx 22,000$ electrons deposited by minimum ionization in a $280 \mu \mathrm{m}$ silicon layer.

\section{Commissioning the Pixel Detector with Cosmic Rays}

Both the barrel and forward detectors were installed in July 2008. Since then, the pixel detectors were included in two data taking commissioning periods, along with the silicon strip detectors. First period was in October - November 2008 [5]. Cosmic muons accumulated in that time were used for the initial alignment of the pixel detector and for the first measurements of the pixel sensor hit efficiency, pixel hit resolution and Lorentz drift angles in barrel and forward detectors. The second data taking period was in July - August 2009. The detector had been moved to work on recovering channels and to improve the cooling system. The alignment procedure [6] as well as the measurements of the efficiency, resolution and Lorentz angles were repeated and found to be consistent with the 2008 results. Data was taken with magnetic field in both zero and 3.8 Tesla configuration.

A total of $\approx 300$ million $(\approx 200$ million) cosmic muon tracks were recorded in 2008 (2009) with the magnetic field turned on. Only $3 \%$ of all tracks that interact with the CMS detector pass through the pixel detector. About 85,000 tracks were reconstructed in the pixel detector in 2008 and more than 65,000 in 2009. 

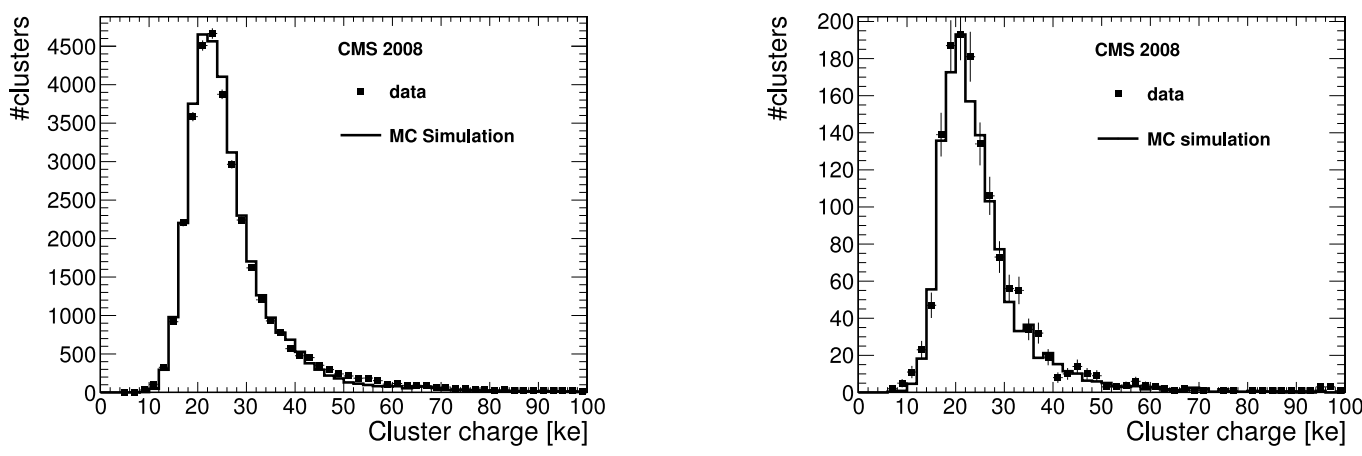

Figure 4: Normalized cluster charge distributions for the barrel (left) and forward (right) detectors.

\subsection{Pixel Detector Status}

Most of pixel detector was operational during both the 2008 and 2009 cosmic ray data taking periods. The fraction of faulty components was very small. There were about $0.9 \%$ (108 out of 11520) barrel ROCs and about 3.1\% (135 out of 4320) forward ROCs which were not operational. Overall 243 out of 15840 (1.5\%) ROCs had problems. The number or dead pixels on an otherwise working ROC was of the order of $0.01 \%$. The noisy pixel rate was of $0.0005 \%$ corresponding to a few hundreds out of 66 million pixels. Noisy pixels were monitored by data quality monitoring system and masked in the data acquisition chain.

\subsection{Cluster Charge}

Tracking particles passing through a pixel layer deposit charge in a few pixels. A collection of adjacent pixels with charge above the readout threshold and with total charge above the cluster threshold forms a cluster. The cluster threshold is about 5000 electrons. Clusters are selected on tracks similar to collision tracks. These tracks pass the following selection criteria. They have an incidence angle on the pixel sensors within $90 \pm 12$ degrees and the transverse momentum is above $4 \mathrm{GeV}$. Only clusters with at least 2 pixels are selected. The cluster charge is normalized so that it corresponds to the charge deposited by tracks with normal incidence with respect to the sensors. The normalization is obtained by multiplying the measured cluster charge by the ratio between the sensor thickness and the track path length through the sensor. The normalized charge distributions for the barrel and forward detectors are shown in figure 4. The Landau peak is at 22,000 electrons as expected for a minimum ionizing particle passing a $285 \mu \mathrm{m}$ silicon layer. The same figure shows that charge distribution shape in data agrees well with the shape in the CMS simulation. The peak in the simulation is shifted with respect to the peak in data by $\approx 1000$ electrons. This shift is due to uncertainties in the calibration which transforms the injected charge from ADC units to electrons. The simulation is corrected for this shift in figure 4. The cluster charge is converted from ADC counts to electrons using the calibration procedure described in section 2.1. The hit position is obtained from the cluster using algorithms described in [7].

\subsection{Measurement of the Lorentz Angle}

In the presence of electric and magnetic fields, the charge carriers freed by a tracking particle 

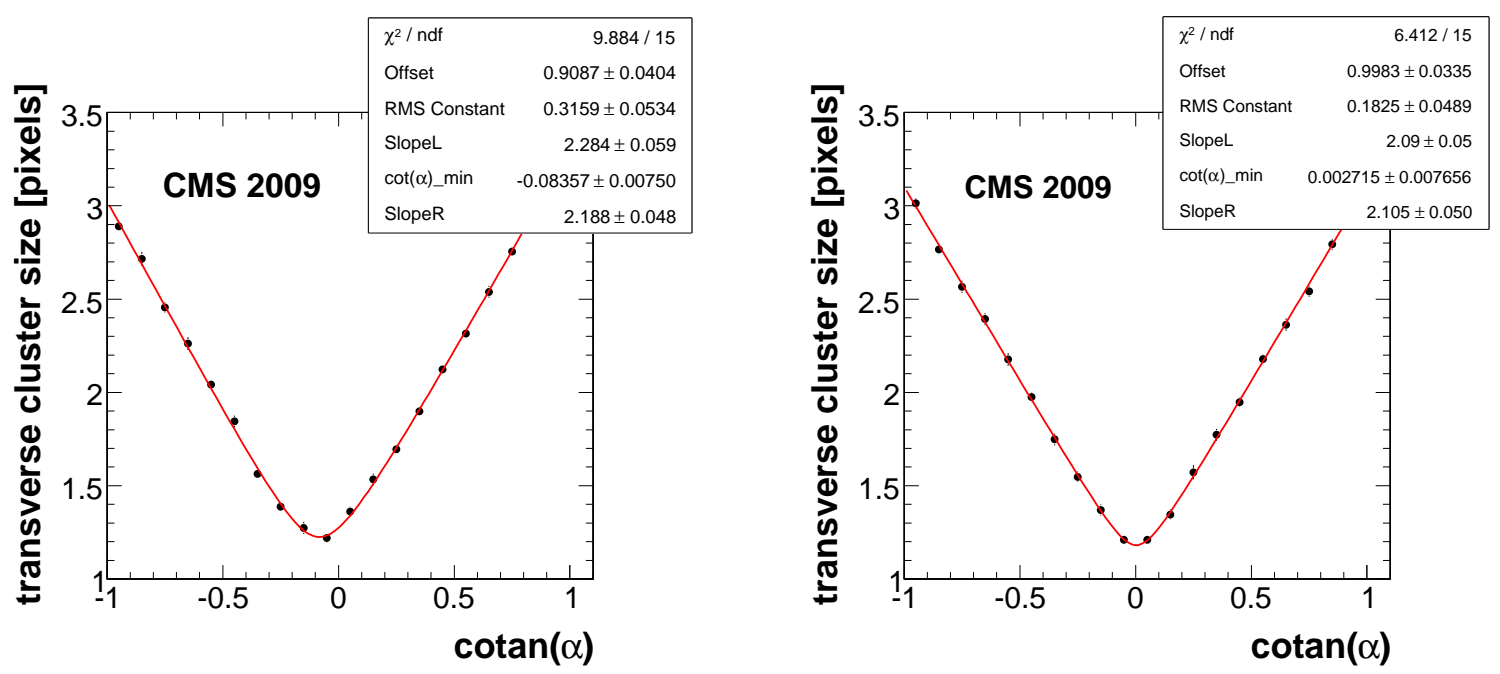

Figure 5: Mean cluster size as function of the cotangent of the incident track angle for the forward detectors with magnetic field turned on at 3.8 Tesla (left) and with the magnetic field turned off (right). In the absence of the magnetic field, the minimum cluster size occurs at normal incidence, consistent with zero Lorentz angle.

are deflected with respect to the electric field due to the Lorentz force. The angle between the electric field and the charge drift direction is called Lorentz angle. The charge drift in the presence of the magnetic field enhances charge sharing. For example, a track perpendicular to the pixel sensor would deposit charge in a single pixel, in the absence of the Lorentz drift. Due to the Lorentz deflection the charge will be collected by more than one pixel and thus improve the resolution. The Lorentz angle is determined using the minimum cluster size method. This method uses the fact that the size of the cluster is minimum for tracks passing through the sensor along Lorentz drift direction. Figure 5 shows the mean cluster size as a function of the cotangent of the incident track angle along the direction perpendicular to the electric and magnetic fields for the forward detector.

From the position of the minima in the cluster size versus incident angle dependence, the Lorentz angles for the barrel and forward detectors are extracted $\theta_{L A}^{\text {barrel }} \approx 22^{\circ}$ and $\theta_{L A}^{\text {forward }} \approx 5^{\circ}$. These results are in very good agreement with the CMS pixel simulation [8].

\subsection{Pixel Sensor Efficiency}

The pixel sensor efficiency is measured by extrapolating tracks from a certain tracking layer to the next. A hit is searched for in a window compatible with the expected track direction. If a hit is found in the expected region, it is added to the track and the track is refit including this new hit. In this case the hit is called valid. If no hit is found in the expected window an invalid or missing hit is added to the track. The pixel sensor efficiency is defined as the ratio between the number of valid hits and the total number of valid and missing hits: $\varepsilon=\sum$ valid $/\left(\sum\right.$ valid $+\sum$ missing $)$. Figure 6 shows the pixel hit efficiency for each ladder and module on each of the three barrel layers. Excluding known dead modules, the pixel efficiency is found to be close to $99 \%$. For the forward detector the efficiency measurement could no be performed because the number of tracks passing through the forward disks was very small. This is due to the specific forward geometry and 

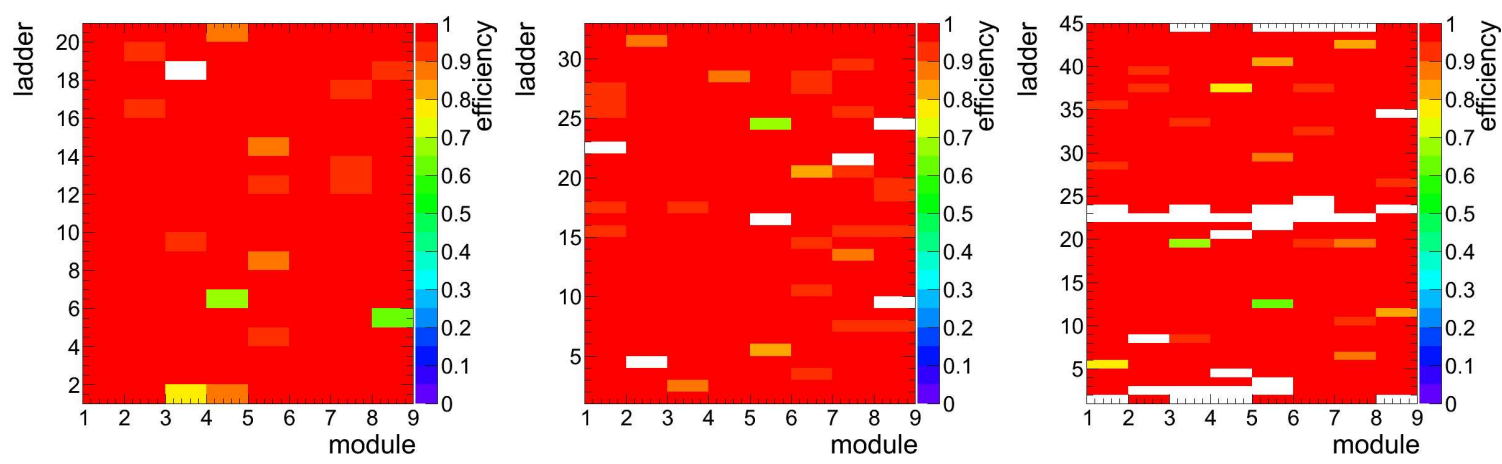

Figure 6: Pixel sensor efficiency for each barrel layer divided into ladders and modules. The white cells are modules which are either dead or the number of pixel hits in these modules was too small to perform the efficiency measurement.

the fact that cosmic tracks tend to be parallel to the forward disks.

\subsection{Pixel Hit Resolution}

The pixel hit resolution is measured using the double-difference method. This method uses tracks that pass through the overlapping regions of two adjacent modules in the same barrel layer. Since the two overlapping modules are very close to each other, extrapolation errors in the determination of the expected hit positions are suppressed. The double difference is calculated as: $\Delta=\Delta x^{\text {reco }}-\Delta x^{\text {pred }}$, where $\Delta x^{\text {reco }}=x_{1}^{\text {reco }}-x_{2}^{\text {reco }}$ and $\Delta x^{\text {pred }}=x_{1}^{\text {pred }}-x_{2}^{\text {pred }}$, with $x_{1,2}^{\text {reco }}$ and $x_{1,2}^{\text {pred }}$ being the reconstructed and expected hit positions in the two modules, respectively. The resolution in the $r \phi$ direction is $19 \pm 2 \mu \mathrm{m}$ and the $z$ direction is $31 \pm 3 \mu \mathrm{m}$. These measurements are in good agreement with the CMS simulation. It is noteworthy that this resolution measurement is done with a very particular set of cosmic tracks that pass through overlapping modules. Since the hit resolution is angle dependent and this track sample includes a very large angular range, the measured resolution is different that the average resolution expected with collision tracks. For collision tracks the expected average resolution is $\approx 12 \mu \mathrm{m}$ for the barrel detector and $\approx 28 \mu \mathrm{m}$ for the forward detector.

\section{Conclusions}

The CMS Pixel detector was installed and commissioned using cosmic rays. About $99 \%$ of channels are operational with very few noisy or dead pixels. The pixel hit efficiency is close to $99 \%$. The pixel hit resolution was measured and found to be in good agreement with expectations from simulations. The 2008 and 2009 cosmic ray data taking periods were extremely useful in establishing and validating calibration procedures like charge calibration and Lorentz angle measurements.

\section{References}

[1] CMS Collaboration, J. Inst. 3 (2008) S08004. 
[2] L. Evans, P. Bryant eds., J. Inst. 3 (2008) S08001.

[3] CMS Collaboration, CMS Physics TDR 8.1 Volume I: Detector Performance and Software, CERN/LHCC 2006-001.

[4] H.C. Kastli et al., Nucl. Instrum. Meth. A 565 (206) 303.

[5] CMS Collaboration, J. Inst. 5 (2010) T03007.

[6] CMS collaboration, J. Inst. 5 (2010) T03009.

[7] M. Swartz et al., Proceedings of Science (Vertex 2007) 035.

[8] V. Chiochia et al., IEEE Trans. Nucl. Sci. 52 (2005) 1067; M. Swartz et al., Nucl. Instrum. Meth. A 565 (2006) 212. 